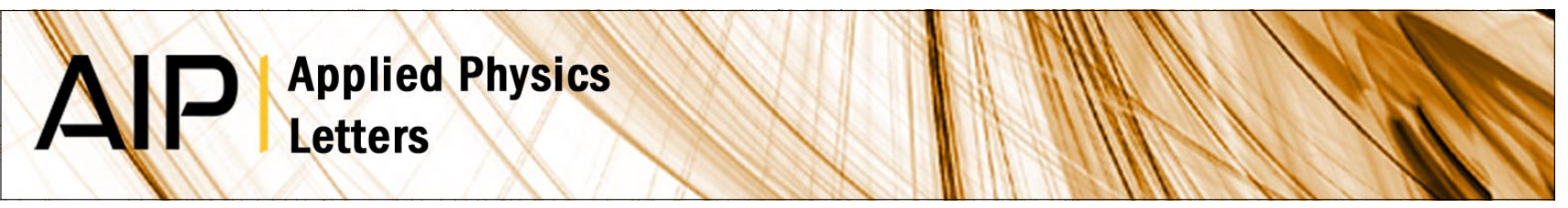

\title{
Temperature dependence of the gain peak in p-doped InAs quantum dot lasers
}

M. Hutchings, I. O'Driscoll, P. M. Smowton, and P. Blood

Citation: Appl. Phys. Lett. 99, 151118 (2011); doi: 10.1063/1.3652702

View online: http://dx.doi.org/10.1063/1.3652702

View Table of Contents: http://apl.aip.org/resource/1/APPLAB/v99/i15

Published by the American Institute of Physics.

\section{Related Articles}

Electroluminescence from strained germanium membranes and implications for an efficient Si-compatible laser Appl. Phys. Lett. 100, 131112 (2012)

A weakly coupled semiconductor superlattice as a potential for a radio frequency modulated terahertz light emitter

Appl. Phys. Lett. 100, 131104 (2012)

Quantum-dot nano-cavity lasers with Purcell-enhanced stimulated emission

Appl. Phys. Lett. 100, 131107 (2012)

Effect of internal optical loss on the modulation bandwidth of a quantum dot laser

Appl. Phys. Lett. 100, 131106 (2012)

Design of three-well indirect pumping terahertz quantum cascade lasers for high optical gain based on nonequilibrium Green's function analysis

Appl. Phys. Lett. 100, 122110 (2012)

\section{Additional information on Appl. Phys. Lett.}

Journal Homepage: http://apl.aip.org/

Journal Information: http://apl.aip.org/about/about_the_journal

Top downloads: http://apl.aip.org/features/most_downloaded

Information for Authors: http://apl.aip.org/authors

\section{ADVERTISEMENT}

\section{(e) ACCELERATE AMBER AND NAMD BY $5 X$. NVIIIA TRYIT ONA FREE, REMOTELYYHOSTED CLUSTER.}




\title{
Temperature dependence of the gain peak in p-doped InAs quantum dot lasers
}

\author{
M. Hutchings, I. O'Driscoll, ${ }^{\text {a) }}$ P. M. Smowton, and P. Blood \\ School of Physics and Astronomy, Cardiff University, Queens Buildings, The Parade, Cardiff, CF24 3AA, \\ United Kingdom
}

(Received 3 August 2011; accepted 26 September 2011; published online 14 October 2011)

\begin{abstract}
Gain peak shifts with injection in undoped and p-doped InAs quantum dot laser structures between $200 \mathrm{~K}$ and $350 \mathrm{~K}$ are measured. The blue-shift with increasing injection, due to state-filling of the inhomogeneous distribution, is temperature independent for a fixed peak gain in the undoped sample, but temperature dependent in the doped sample. This is due to the wide electron state distribution and lowering of the electron quasi Fermi level by p-doping relative to the undoped device. While p-doping reduces the temperature dependence of the threshold current, it comes at the expense of increasing the temperature sensitivity of the wavelength. (C) 2011 American Institute of Physics. [doi:10.1063/1.3652702]
\end{abstract}

The optical spectra of self-assembled quantum dot lasers are broadened by the distribution of dot sizes ${ }^{1}$ and by homogeneous broadening; this brings disadvantages such as an intrinsic temperature dependence of threshold current ${ }^{2}$ and benefits such as a broad gain spectrum with potential for short pulse generation. ${ }^{3}$ A further consequence of the inhomogeneous energy distribution of dot states is that when their occupation is determined by a global Fermi function, the peaks of the emission and gain spectra shift with temperature and with drive current, akin to band-filling in quantum well and bulk materials. The gain peak position is of particular importance for quantum dot vertical cavity lasers, which are of current interest, ${ }^{4}$ where the gain spectrum should be matched to the reflection spectrum of the mirrors. In this paper, we report an experimental study of state filling in quantum dot laser structures which we interpret using model calculations. P-doping has been shown to modify the temperature dependence of threshold ${ }^{5}$ and the modulation response ${ }^{6}$ of quantum dot lasers, therefore, this work is concerned particularly with a comparison between nominally undoped and p-doped structures, and the findings are relevant to the design of quantum dot lasers particularly where the gain peak and its temperature dependence are important.

We have studied two $1.3 \mu \mathrm{m}$ laser structures with five layers of dots with "high growth temperature spacer layers"7 and having nominally identical growth conditions except that in the active region one is nominally undoped, while the other has Be p-dopant in $6 \mathrm{~nm}$ of the $50 \mathrm{~nm}$ GaAs spacer between the dot layers. Atomic force microscopy measurements were used to determine the dot density and suggest there are about 15 dopant atoms per dot. Oxide-insulated, $50 \mu \mathrm{m}$ wide stripe, segmented contact devices with $300 \mu \mathrm{m}$ long sections were fabricated and modal gain and absorption spectra were measured using pulsed electrical excitation by observation of the single-pass amplified spontaneous emission. ${ }^{8}$

Gain spectra for the doped structure at $300 \mathrm{~K}$ for various drive currents are shown in Fig. 1, together with the absorp-

a)Electronic mail: odriscollip@cardiff.ac.uk. tion spectrum. The lowest energy gain peaks all occur at a lower energy than that of the first absorption peak $(0.972 \mathrm{eV})$; we associate these peaks with ground state transitions. At the lowest current, the gain peak is $18 \mathrm{meV}$ below the absorption peak, then, as the current is increased, the gain peak first moves to higher energy, closer to the absorption peak, before shifting back to lower energy at the highest injection. This latter behavior is due to many-body Coulomb interactions, 9,10 while we associate the shift to higher energy at lower current with the filling of states in the inhomogeneous distribution. Qualitatively similar behavior is observed in the un-doped sample.

To compare the doped and undoped samples, it is necessary to identify an independent measure of the degree of inversion of the system. The experimental drive current density includes unknown contributions from non-radiative processes, which may also be different in the two samples. Neither is it appropriate to use the quasi-Fermi level

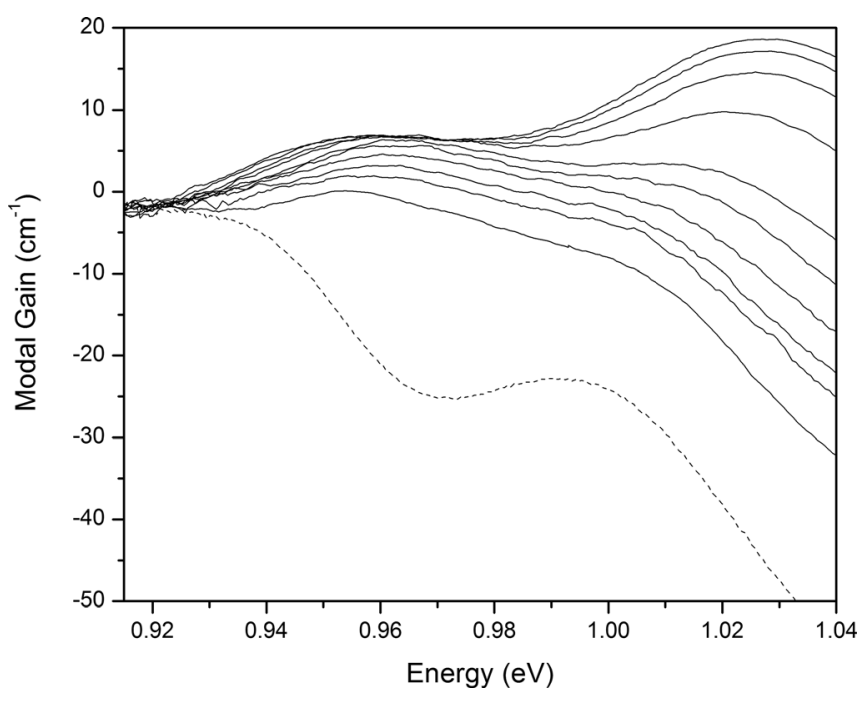

FIG. 1. Modal gain spectra (solid lines) over a range of injection currents: $15 \mathrm{~mA}, 20 \mathrm{~mA}, 25 \mathrm{~mA}, 30 \mathrm{~mA}, 40 \mathrm{~mA}, 50 \mathrm{~mA}, 80 \mathrm{~mA}, 140 \mathrm{~mA}, 180 \mathrm{~mA}$, and $200 \mathrm{~mA}$ along with the modal absorption spectrum (dashed line) for the p-doped sample at $300 \mathrm{~K}$. 


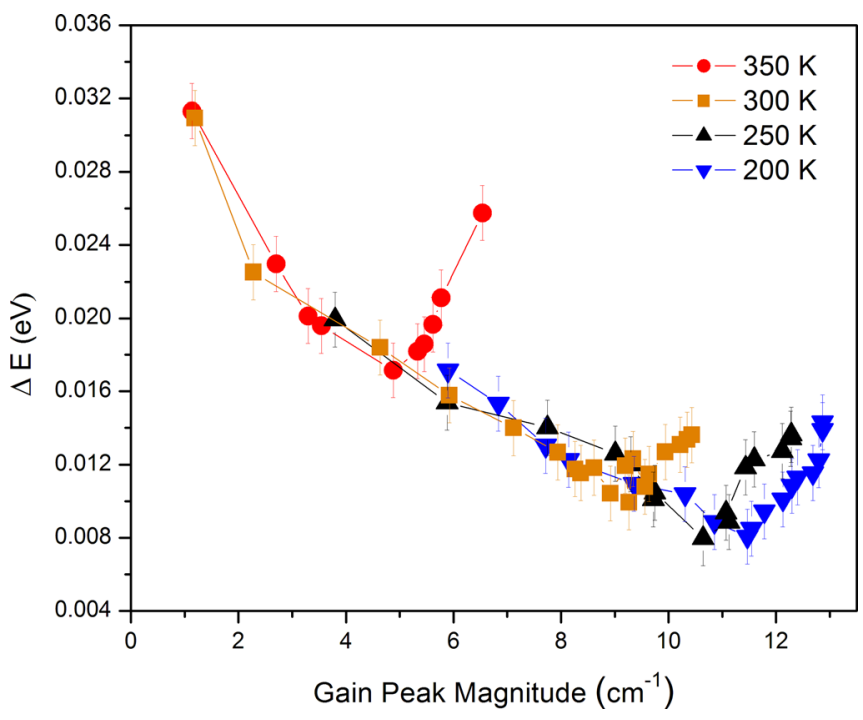

FIG. 2. (Color online) Plot of the experimentally measured modal absorption peak energy minus gain peak energy, $\Delta \mathrm{E}$, versus gain peak magnitude for the un-doped sample at $350 \mathrm{~K}, 300 \mathrm{~K}, 250 \mathrm{~K}$, and $200 \mathrm{~K}$.

separation obtained from the transparency photon energy because this includes injection dependent shifts by (unknown) "band gap narrowing." We have chosen to use the magnitude of the ground state peak gain as a proxy for the degree of inversion since it is proportional to the difference in electron occupation probabilities of upper and lower states at the gain peak. We plot the difference between the energy of the gain peak and the energy of the absorption peak for each sample as a function of the magnitude of the ground state peak gain, with the results shown in Figs. 2 and 3 for measurements between $200 \mathrm{~K}$ and $350 \mathrm{~K}$. These figures show similar qualitative trends as noted above: the gain peak first shifts toward the absorption peak (smaller $\Delta \mathrm{E}$ ) with increasing current, then shifts back to lower energy at the highest currents. However, the behavior with temperature is very different for the doped and un-doped structures. In the un-doped sample, the blue-shift due to state filling is

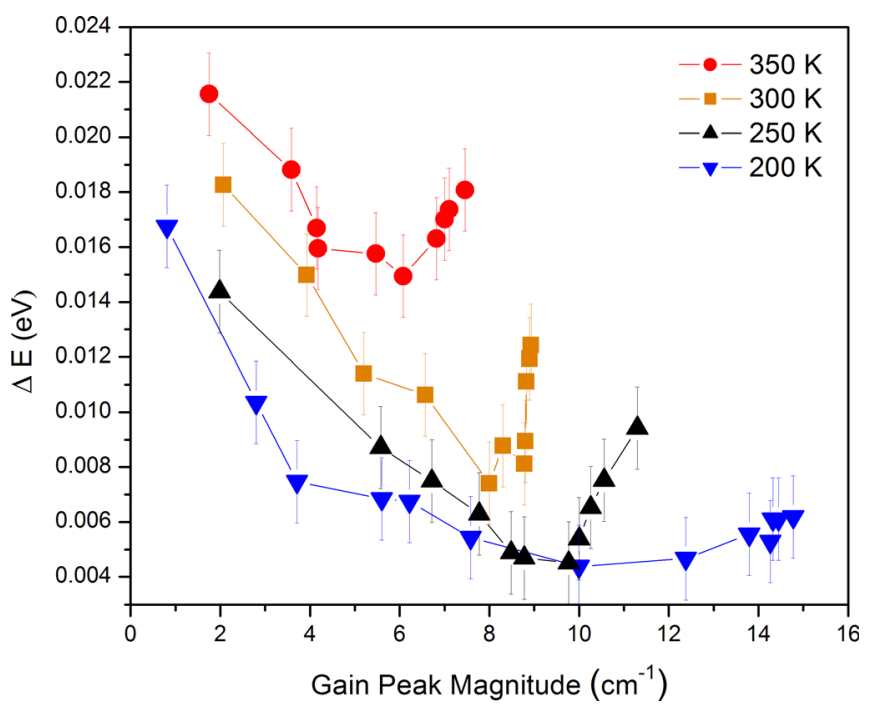

FIG. 3. (Color online) Plot of the experimentally measured modal absorption peak energy minus gain peak energy, $\Delta \mathrm{E}$, versus gain peak magnitude for the p-doped sample at $350 \mathrm{~K}, 300 \mathrm{~K}, 250 \mathrm{~K}$, and $200 \mathrm{~K}$. the same at all temperatures for a given peak gain, whereas for the p-doped sample, the shift due to state filling is different at different temperatures: for the p-doped sample, the gain peak energy is temperature dependent at any given fixed gain.

To understand the origin of this behavior, we have calculated the Fermi-Dirac carrier distributions across the electron and hole ground states and the wetting layer of an inhomogeneous dot distribution assuming charge neutrality of the whole system. The energy states are calculated for harmonic potentials and we construct an inhomogeneous Gaussian distribution ${ }^{11}$ of 51 transition energies by setting appropriate values for the proportionality constant which defines the potentials. The calculation of absorption and gain also incorporates the homogeneous linewidth. The inhomogenity input to the model is then adjusted until the calculated spectrum matches the experimentally obtained modal absorption spectrum; therefore, this comparison includes both the effects of inhomogeneous and homogeneous broadening. The calculation also incorporates a temperature (a)
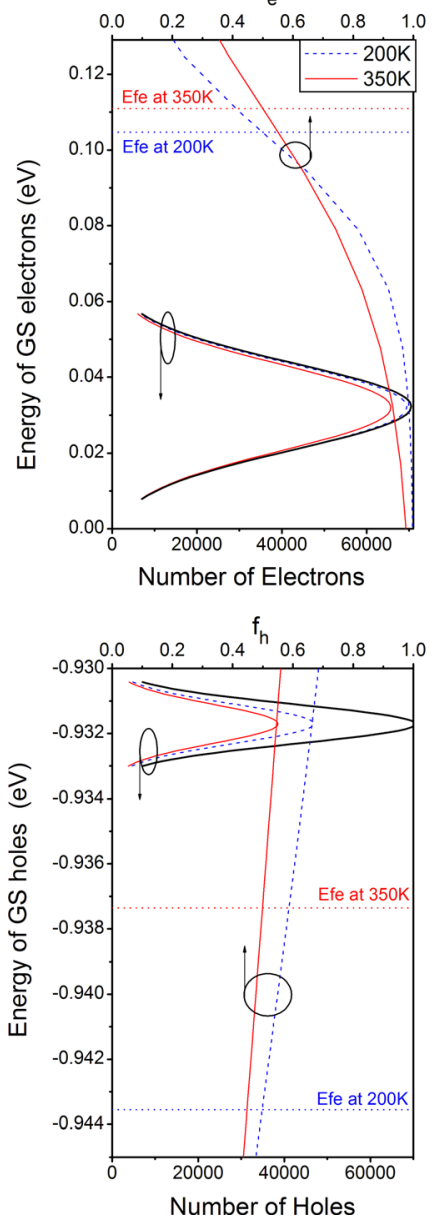

(b)

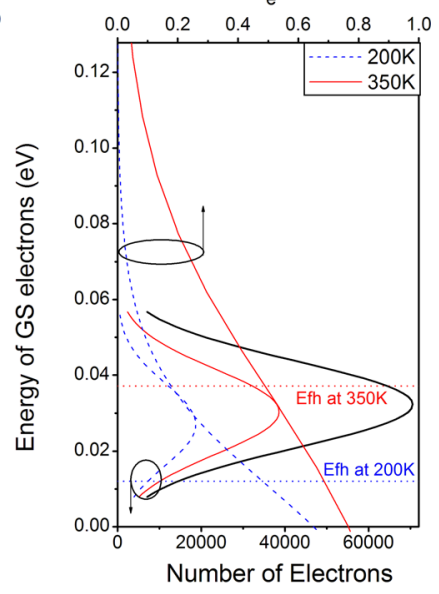

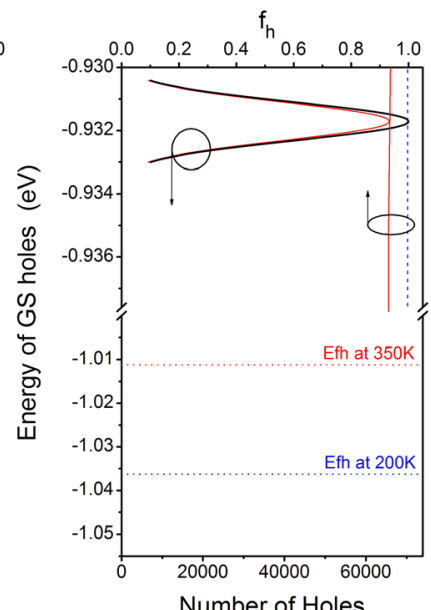

FIG. 4. (Color online) Ground state (GS) distributions of the un-doped (a) and doped (b) samples are shown as a function of energy, relative to the conduction band edge in the dots, for the electrons (top) and holes (bottom). The thick solid line shows the inhomogeneous state distribution. The distribution of occupied electron/holes is shown by the solid and dashed lines at $350 \mathrm{~K}$ and $200 \mathrm{~K}$, respectively, for a fixed quasi-Fermi level separation of $1.0483 \mathrm{eV}$ as well as the calculated occupation probabilities (upper $\mathrm{X}$-axis). The quasi-Fermi level energies $\left(\mathrm{Ef}_{\mathrm{e}}\right.$ and $\mathrm{Ef}_{\mathrm{h}}$ ) for each temperature are indicated by the dotted lines. 
dependent linewidth taken from Ref. 12. The full width at half maximum of the input inhomogeneous electron and hole state distributions required to fit the absorption spectra were $27 \mathrm{meV}$ and $1.4 \mathrm{meV}$, respectively, using effective masses for electrons and holes of $0.027 \mathrm{~m}_{0}$ and $0.34 \mathrm{~m}_{0}$. In the p-doped structure, the ionization energy for Be acceptors was taken to be $28 \mathrm{meV}$.

Fig. 4(a) shows the available ground state inhomogeneous distributions in energy of the electron (top) and hole (bottom) states used in the calculation, and the electron and hole occupation probabilities $\left(f_{e}\right.$ and $f_{h}$ ), at $200 \mathrm{~K}$ and $350 \mathrm{~K}$ for the same quasi-Fermi level separation of $1.0483 \mathrm{eV}$, chosen to be greater than the gain peak energy and, therefore, typical of the quasi-Fermi level separation when the system is inverted. (In this calculation at fixed quasi-Fermi level separation, the occupation probability of the electron states goes down with increasing temperature because they are located below the quasi-Fermi energy.) The figure also shows the carrier distributions which arise from the product of the state distributions and the occupation probability. The electron states are almost fully occupied and the distributions follow the inhomogeneous state distributions with very little shift in their peak positions with temperature and this is consistent with the experimental gain data in Fig. 2.

The calculated data for the p-doped sample in Fig. 4(b), for the same state distributions, show that doping lowers the quasi-Fermi level positions relative to the undoped sample with the result that the electron states are only partially occupied, being in the tail of the Fermi distribution, and the peak of the electron distribution at fixed gain shifts with temperature. The separation between the peaks of the electron and hole distributions increases by $5 \mathrm{meV}$, going from $200 \mathrm{~K}$ to $350 \mathrm{~K}$, at fixed quasi-Fermi level separation for this simple model system and this will cause a shift in the energy of the gain peak. This shift of the gain peak for fixed gain which we observe experimentally is about $8 \mathrm{meV}$ (Fig. 3).

There are two origins to the distinct behaviour of the p-doped sample. First, the electron state distribution has a width at half height of about $\mathrm{k}_{\mathrm{B}} \mathrm{T}$ at room temperature, whereas the hole distribution is narrower, much less than $k_{B} T$. Consequently, the distribution of holes follows the inhomogeneous state distribution and does not shift with temperature in either case (Fig. 4). The second factor is the effect of doping on the electron quasi-Fermi level. In the undoped sample, the electron quasi-Fermi level is higher than the electron state distribution (Fig. 4(a)), and most electron states are filled and the electron distribution follows the state distribution and is not temperature dependent. However, the electron quasi-Fermi level in the doped sample is at lower energy than the electron states (Fig. 4(b)), their occupation probability is low (0.2-0.4), and the electron distribution is sensitive to the Fermi factor and shifts with temperature as is apparent in Fig. 4(b). Thus, it is the combination of a wide electron state distribution and the lowering of the electron quasi Fermi level by doping, which results in the temperature sensitivity of the electron distribution and the gain peak in the presence of doping.

In summary, we have shown that the blue-shift of the gain peak due to state-filling in un-doped quantum dot structures is independent of temperature at a given value of peak gain, and over the temperature range studied $(200 \mathrm{~K}$ to $350 \mathrm{~K}$ ) whereas in structures with about 15 acceptors per dot in the spacer layers, the state filling is temperature dependent at any fixed gain. This behavior is a consequence of the wide electron state distribution and the lowering of the electron quasi Fermi level by p-doping relative to the un-doped device. Thus, in p-doped dots, the behavior of the gain peak with temperature and injection is more complex than in undoped structures, requiring additional care in the detailed design of devices. While p-doping is often introduced to reduce the temperature dependence of the threshold current, it comes at the expense of increasing the temperature sensitivity of the lasing wavelength.

The authors would like to thank H. Y. Liu and M. Hopkinson for growing the material used in this work. We acknowledge financial support from the Engineering and Physical Sciences Research Council under Grant No. EP/F006683.

\footnotetext{
${ }^{1}$ N. Ledentsov, M. Grundmann, F. Heinrichsdorff, D. Bimberg, V. M. Ustinov, A. E. Zhukov, M. V. Maximov, Zh. I. Alferov, and J. A. Lott, IEEE J. Sel. Top. Quantum Electron. 6, 439 (2000).

${ }^{2}$ A. E. Zhukov, V. M. Ustinov, A. Y. Egorov, A. R. Kovsh, A. F. Tsatsul'nikov, N. N. Ledentsov, S. V. Zaitsev, N. Y. Gordeev, P. S. Kop'ev, and Z. I. Alferov, Jpn. J. Appl. Phys. 1(36), 4216 (1997).

${ }^{3}$ M. Kuntz, G. Fiol, M. Laemmlin, C. Meuer, and D. Bimberg, Proc. IEEE 95, 1767 (2007).

${ }^{4}$ J. A. Lott, V. A. Shchukin, N. N. Ledentsov, A. M. Kasten, and K. D. Choquette, Electron. Lett. 47, 12 (2011).

${ }^{5}$ P. M. Smowton, I. C. Sandall, D. Mowbray, and H. Liu, IEEE J. Sel. Top. Quantum Electron. 13, 1261 (2007).

${ }^{6}$ D. G. Deppe, H. Huang, and O. B. Shchekin, IEEE J. Quantum Electron. 38, 1587 (2002).

${ }^{7}$ H. Y. Liu, I. R. Sellers, T. Badcock, D. J. Mowbray, and M. Skolnick, Appl. Phys. Lett. 85, 704 (2004).

${ }^{8}$ P. Blood, G. Lewis, P. M. Smowton, and H. Summers, IEEE J. Sel. Top. Quantum Electron. 9, 1275 (2003).

${ }^{9}$ I. O'Driscoll, M. Hutchings, P. M. Smowton, and P. Blood, Appl. Phys. Lett. 97, 141102 (2010).

${ }^{10}$ H. C. Schneider and W. W. Chow, Phys. Rev. B 64, 115315 (2001).

${ }^{11}$ I. O'Driscoll, P. Blood, and P. M. Smowton, IEEE J. Quantum Electron. 46, 525 (2010).

${ }^{12}$ P. Borri, W. Langbein, S. Schneider, U. Woggon, R. L. Sellin, D. Ouyang,
} and D. Bimberg, IEEE J. Sel. Top. Quantum Electron. 8, 984 (2002). 